\title{
The reliability of vertical jump tests between the Vertec and My Jump phone application
}

\author{
Vanessa R Yingling Corresp., ${ }^{1}$ ， Dimitri A Castro ${ }^{1}$ ， Justin T Duong ${ }^{1}$ ， Fiorella J Malpartida ${ }^{1}$ ， Justin R Usher ${ }^{1}$, \\ Jenny $\mathrm{O}^{1}$ \\ ${ }^{1}$ Department of Kinesiology, California State University, East Bay, Hayward, California, United States \\ Corresponding Author: Vanessa R Yingling \\ Email address: vanessa.yingling@csueastbay.edu
}

Background. The vertical jump is used to estimate sports performance capabilities and physical fitness in children, elderly, non-athletic and injured individuals. Different jump techniques and measurement tools are available to assess vertical jump height and peak power; however, their use is limited by access to laboratory settings, excessive cost and/or time constraints thus making these tools oftentimes unsuitable for field assessment. A popular field test uses the Vertec and the Sargent vertical jump with countermovement; However, new low cost, easy to use tools are becoming available, including the $M y$ Jump iOS mobile application (app).The purpose of this study was to assess the reliability of the My Jump relative to values obtained by the Vertec for the Sargent stand and reach vertical jump (VJ) test.

Methods. One-hundred-and-thirty-five healthy participants aged 18-39 years (94 males, 41 females) completed three maximal Sargent VJ with countermovement that were simultaneously measured using the Vertec and the My Jump. Jump heights were quantified for each jump and peak power was calculated using the Sayers equation. Four separate ICC estimates and their 95\% confidence intervals were used to assess reliability. Two analyses (with jump height and calculated peak power as the dependent variables, respectively) were based on a single rater, consistency, 2-way mixed-effects model, while two others (with jump height and calculated peak power as the dependent variables, respectively) were based on a single rater, absolute-agreement, 2-way mixed-effects model.

Results. Moderate to excellent reliability relative to the degree of consistency between the Vertec and My Jump values was found for jump height (ICC $=0.813 ; \mathrm{Cl} 95 \%=.747-.863)$ and calculated peak power (ICC $=.926 ; \mathrm{Cl} 95 \%=.897-.947)$. However, poor to good reliability relative to absolute agreement for VJ height (ICC $=.665 ; 95 \% \mathrm{Cl}=.050-.859)$ and poor to excellent reliability relative to absolute agreement for peak power (ICC =.851; Cl 95\% .272-.946) between the Vertec and My Jump values were found; Vertec VJ height, and thus, Vertec calculated peak power values, were significantly higher than those calculated from My Jump values $(p<.0001)$.

Discussion. The My Jump app may provide a reliable measure of vertical jump height and calculated peak power in multiple field and laboratory settings without the need of costly equipment such as force plates or Vertec. The reliability relative to degree of consistency between the Vertec and My Jump app was moderate to excellent. However, the reliability relative to absolute agreement between Vertec and My Jump values contained significant variation (based on $\mathrm{Cl}$ values), thus, it is recommended that either the My Jump or the Vertec be used to assess VJ height in repeated measures within-subjects' designs; these measurement tools should not be considered interchangeable within subjects or in group measurement designs. 
1

2 The reliability of vertical jump tests between the Vertec and My Jump phone application

3

4 Vanessa R Yingling, Dimitri A Castro, Justin T Duong, Fiorella J Malpartida, Justin R Usher,

5 Jenny $\mathrm{O}$

6

7 Department of Kinesiology, California State University, East Bay, Hayward, California, United

8 States of America

9

10

11 Corresponding Author:

12 Vanessa R Yingling

13 Department of Kinesiology

1425800 Carlos Bee Boulevard

15 Hayward, CA 94542

16 vanessa.yingling@csueastbay.edu

17 


\section{Abstract (452 words total)}

Background. The vertical jump is used to estimate sports performance capabilities and physical fitness in children, elderly, non-athletic and injured individuals. Different jump techniques and measurement tools are available to assess vertical jump height and peak power; however, their use is limited by access to laboratory settings, excessive cost and/or time constraints thus making these tools oftentimes unsuitable for field assessment. A popular field test uses the Vertec and the Sargent vertical jump with countermovement; However, new low cost, easy to use tools are becoming available, including the My Jump iOS mobile application (app). The purpose of this study was to assess the reliability of the My Jump relative to values obtained by the Vertec for the Sargent stand and reach vertical jump (VJ) test.

Methods. One-hundred-and-thirty-five healthy participants aged 18-39 years (94 males, 41 females) completed three maximal Sargent VJ with countermovement that were simultaneously measured using the Vertec and the My Jump. Jump heights were quantified for each jump and peak power was calculated using the Sayers equation. Four separate ICC estimates and their 95\% confidence intervals were used to assess reliability. Two analyses (with jump height and calculated peak power as the dependent variables, respectively) were based on a single rater, consistency, 2-way mixed-effects model, while two others (with jump height and calculated peak power as the dependent variables, respectively) were based on a single rater, absolute-agreement, 2-way mixed-effects model.

Results. Moderate to excellent reliability relative to the degree of consistency between the Vertec and My Jump values was found for jump height (ICC $=0.813$; CI 95\% $=.747-.863$ ) and calculated peak power (ICC $=.926$; CI 95\% $=.897-.947)$. However, poor to good reliability relative to absolute agreement for $\mathrm{VJ}$ height $(\mathrm{ICC}=.665 ; 95 \% \mathrm{CI}=.050-.859)$ and poor to excellent reliability relative to absolute agreement for peak power (ICC $=.851$; CI 95\% .272.946) between the Vertec and My Jump values were found; Vertec VJ height, and thus, Vertec calculated peak power values, were significantly higher than those calculated from My Jump values $(p<.0001)$.

Discussion. The My Jump app may provide a reliable measure of vertical jump height and calculated peak power in multiple field and laboratory settings without the need of costly equipment such as force plates or Vertec. The reliability relative to degree of consistency between the Vertec and My Jump app was moderate to excellent. However, the reliability relative to absolute agreement between Vertec and My Jump values contained significant variation (based on CI values), thus, it is recommended that either the My Jump or the Vertec be used to assess VJ height in repeated measures within-subjects' designs; these measurement tools should not be considered interchangeable within subjects or in group measurement designs. 
60

61 Introduction

62

63 Vertical jump height is a measurement that coaches, physical educators, health care

64 professionals, and strength and conditioning practitioners use to predict or assess physical

65 performance for talent identification and player development purposes. For example, many sport 66

67 (Teramoto, Cross \& Willick, 2016). Moreover, the literature has demonstrated that individual 68

and sport characteristics such as sex, skill level, sport position, and risk of injury are associated with vertical jump performance (Burr et al., 2008; Mujika et al., 2009; Marques \& Izquierdo, 2014; Janot, Beltz \& Dalleck, 2015; Spiteri et al., 2017). Vertical jump performance has also been found to be associated with neuromuscular fatigue and thus has been used to monitor and avoid overtraining in athletes (Gathercole et al., 2015). Lastly, vertical jump tests correlate with total and lower extremity lean mass (Stephenson et al., 2015) and bone strength (Janz et al., 2015; Yingling et al., 2017). The "gold standards" for vertical jump height measurement are video analysis to calculate the position of the body's center of mass (Aragón, 2000) and integration of the ground reaction force measured on a force plate (Menzel et al., 2010). However, relative to "real-world" assessment by non-elite and/or non-research populations, limited access to laboratory settings, excessive cost of such measurement tools, time, and/or expertise constraints render these approaches largely unsuitable for field assessments conducted by many sport and physical activity practitioners.

Many devices have been developed to measure vertical jump height in a low cost and reliable manner, including contact mats (Just Jump System, Ergo Jump), velocity systems (GymAware, accelerometers), and linear position transducers (OptoJump, Myotest, Vertec). Three factors can 
85 affect the reliability and validity of all these approaches: the method used to calculate height, the 86 type of jump performed, and body mass. The force plate, considered the "gold standard",

87 measures jump height by calculating flight time of the jump (Walsh et al., 2006; Glatthorn et al., 88 2011); however, excessive hip and/or knee flexion during the jump can overestimate flight time 89 and jump height (Nuzzo, Anning, \& Scharfenberg, 2011). The type of jump used to assess 90 subjects has varied between studies and was typically dependent on the purpose of the 91 assessment, the population assessed, and the setting of the assessment. The squat jump (SJ) and 92 countermovement jump (CMJ) are predominantly used in laboratory settings (Markovic et al., 93 2004; Nuzzo, Anning, \& Scharfenberg, 2011), but a common field test used in physical 94 education settings as well as in professional sport combines is the Sargent jump and reach test 95 (VJ) (de Salles et al., 2012; Castagna et al., 2013; Ayán-Pérez et al., 2017). The VJ is not only 96 focused on the lower limbs but necessitates coordination of both the lower and upper limbs 97 (Markovic et al., 2004; Leard et al., 2007; Nuzzo, Anning \& Scharfenberg, 2011) as the upper 98 limbs may increase take-off velocity up to 10\% (Luhtanen \& Komi, 1978; Harman et al., 1988). 99 High reliability of the jump and reach test was reported in both pre-school age children (Ayán100 Pérez et al., 2017) and in athletes (14 year old soccer players) (de Salles et al., 2012). In 101 addition, ecological validity was found for VJ testing in activities such as basketball and 102 volleyball, in which reaching height is key during the jump (Menzel et al., 2010). A common 103 measuring tool of the VJ in the field is the Vertec, however the Vertec requires execution of a 104 more complex jump from the participant, potentially affecting the reliability of the VJ heights 105 (Buckthorpe, Morris \& Folland, 2012; Harman et al., 1988; Nuzzo, Anning \& Scharfenberg, 106 2011). The participant must be able to coordinate the arm swing such that the arms are fully 107 extended and in contact with the vanes at the moment that the participant has attained their 
108 greatest displacement from the floor (Harman et al., 1988). In addition, measurement error may

109 be introduced due to the 2-step measurement protocol for the Vertec as well as the vane spacing

110 on the Vertec (Nuzzo, Anning \& Scharfenberg, 2011). Yet, the use of the Vertec to measure VJ

111 height remains commonplace in physical education and sport settings due to its convenience and

112 price point. Although the measurement tool and type of jump may introduce error, body mass is

113 also a large factor affecting VJ height (Sayers et al., 1999). The difference in body mass can

114 significantly affect the vertical height reached by two participants however the impulse generated 115 may be similar (Harman et al., 1988).

116

117 A new approach to vertical jump height measurement is the use of mobile applications. My

118 Jump, a mobile application for iOS and android devices, uses the device camera's frame-by-

119 frame analysis to calculate flight time and jump height. Recent studies have found almost perfect 120 agreement between the force plate and My Jump for measuring countermovement jump height 121 using either time in air (Balsalobre-Fernández, Glaister \& Lockey, 2015; Driller et al., 2017) or 122 calculated height from take-off velocity (Carlos-Vivas et al., 2016). Furthermore, excellent 123 agreement between force plate and My Jump measurements was found for three different types 124 of jumps including the countermovement jump (CMJ), squat jump (SJ) and drop jump (DJ) in 125 both male and female competitive athletes (Gallardo-Fuentes et al., 2016; Stanton, Wintour \& 126 Kean, 2017). Intra rater reliability for both CMJ and DJ was also found to be excellent (Stanton, 127 Wintour \& Kean, 2017). My Jump is an affordable, portable alternative relative to other tools 128 that assess vertical jump performance. Moreover, high reliability and accuracy of My Jump 129 compared to the gold standard (force plate) has been reported (Balsalobre-Fernández, Glaister \& 130 Lockey, 2015; Gallardo-Fuentes et al., 2016; Carlos-Vivas et al., 2016; Stanton, Wintour \& 
131 Kean, 2017). However, both the force plate and My Jump use flight time as the source of the

132 height calculation (Balsalobre-Fernández, Glaister \& Lockey, 2015) while the commonly used

133 field measurement is a direct distance measurement of jump height. Thus, the Vertec may yield

134 different absolute jump height values compared to My Jump, yet no studies to date have

135 compared My Jump to the Vertec. Therefore, the primary purpose of the study was to examine

136 the reliability of My Jump VJ values compared to those of Vertec. A secondary purpose was to

137 examine whether the use of raw VJ values versus calculated external peak power values using

138 the Sayers equation influenced reliability results. We hypothesized that: a) reliability relative to

139 degree of consistency between the measurement tools (Vertec and My Jump) would be high, and,

140 b) reliability, in terms of absolute agreement between the measurement tools would be low, with

141 a detectable systematic difference.

142

143 Materials \& Methods

144

145 Correlational study

146

147 Participants

148 One-hundred-and-thirty-five healthy adults (94 males, 41 females; university students, staff, and

149 faculty) participated in the study (Table 1). All participants were informed of the risks and

150 benefits of the study and provided written informed consent. All study procedures were

151 approved by the California State University, East Bay Institutional Review Board (CSUEB-IRB-

152 2015-275-F).

153

154 Experimental Protocol

155 Procedures 
156 Participants completed a general health and demographic survey and were excluded if they had a

157 history of health concerns, a disease or physical condition that may affect physical activity, or,

158 were pregnant. The demographic information collected includes sex, height, and mass. Height

159 and mass were measured using a stadiometer and a calibrated scale. All participants were asked

160 if they were competitive athletes (yes/no; defined as: "One who plays an organized sport for a

161 team or in an organization"), and whether they regularly participated in vigorous physical

162 activity (yes/no; defined as: "Activity that causes large increases in breathing or heart rate for at

163 least 10 minutes continuously").

164

165 Two vertical jump measuring systems, Vertec and My Jump, were used simultaneously to assess

166 VJ height. "Peak Power" was then calculated from the jump height measured from the two

167 measuring systems (Sayers et al., 1999). Jump height was quantified using a Vertec

168 (JUMPUSA.com, Sunnyvale, CA) while also being recorded using an iPad mini 2 (Frame Rate

$16960 \mathrm{fps}, 1080 \mathrm{p}$ video, Apple Inc, USA). The take-off and landing frames from the video were

170 determined using My Jump and flight time (ms) was then calculated. The jump height was

171 determined using the calculation:

172

173 Height $($ meters $)=\left(\mathrm{g}^{*}\right.$ time $\left.^{\wedge} 2\right) / 8$ where $\mathrm{g}=9.81 \mathrm{~m} / \mathrm{s}^{\wedge} 2 \quad($ Bosco, Luhtanen $\&$ Komi, 1983$)$

174

175

176 The iPAD mini 2 was connected to a tripod and placed perpendicular to the frontal plane of the

177 participants focused on their feet and approximately 1.5 meters from the participant. One 
178 researcher was responsible for all analysis of flight time duration; takeoff was determined as the

179 first frame with both feet off the ground and landing when at least on foot touched the ground.

180

181 Participants were given the option to participate in a warm up exercise consisting of 10 squats, 18210 alternating high knees, and one-minute running in place. Following verbal explanation of the 183 jump and reach countermovement jump and a physical demonstration by a research assistant, the 184 participants standing reach height was measured using the Vertec followed by three VJ jumps as 185 high as possible to displace the Vertec vanes. At the moment preceding the jump, the participants 186 could freely flex the hip, knee and ankle joints and prepare the upper limbs for a sudden upward 187 thrust, in an effort to promote the highest vertical jump possible. The rest time between jumps 188 was 20 seconds. The participant's vertical jump height was calculated as the difference between 189 their maximum jump height and standing reach height. "Peak Power" was calculated from the 190 maximal jump height of three trials.

191

192 Sayers Peak Power Equation (Sayers et al., 1999)

193

194 Peak Power $(\mathrm{W})=[51.9 *$ VJ height $(\mathrm{cm})]+[48.9 *$ Body mass $(\mathrm{kg})]-2007$

196 Statistical Analysis:

197

198 Intraclass correlation (ICC) is a measure of reliability which assesses both, degree of correlation 199 (i.e., consistency) and degree of absolute agreement between two variables (Shrout \& Fleiss, 200 1979). Given the purpose of our study, we were equally interested in consistency and absolute 
201 agreement between the two measurement tools. We were also interested in determining whether

202 VJ jump height and Peak Power (calculated using the Sayer's (1999) equation) produced

203 differential ICC results. As such, reliability was assessed using four separate ICC estimates and

204 their 95\% confidence intervals (calculated using SPSS statistical package version 23; SPSS Inc.,

205 Chicago, IL). More specifically, we conducted four separate single-rater two-way random

206 effects model ICCs. Two analyses (with jump height and Peak Power as the dependent variables,

207 respectively) were based on a single rater, consistency, two-way random-effects model, while

208 two others (with jump height and Peak Power as the dependent variables, respectively) were

209 based on a single rater, absolute-agreement, two-way random-effects model. A two-way random

210 effects model is noted to be appropriate for evaluating assessment methods that are intended for

211 routine use by raters with similar characteristics (Koo \& Li, 2016). We chose a single rater ICC

212 type as we assumed a single rater would be the basis for real world measurement of jump height

213 (e.g., a single coach, trainer, PE teacher, etc. will administer the vertical jump test during

214 assessment). We chose to adopt Koo and Li's guidelines for interpretation of ICC values; based

215 on a confidence interval (CI) of $95 \%$ of the ICC estimate, $<0.50,0.50-0.75,0.75-0.90$, and $>0.90$

216 represent poor, moderate, good, and excellent ICC, respectively (Koo \& Li, 2016). Analysis

217 separating the participants by sex was also run. Paired student's t-test were performed to

218 determine any systematic differences between the absolute values of jump height between the

219 two measurement tools, Vertec and My Jump. Bland-Altman plots were constructed using Graph

220 Pad (GraphPad Prism version 6.00 for Windows, GraphPad Software, San Diego, California,

221 USA).

222

223 Results

224

225 Consistency 
227 The ICC estimate and 95\% CI demonstrated good reliability for jump height (ICC $=.813 ; 95 \%$

$228 \mathrm{CI}=.747-.863)$ and excellent reliability for calculated Peak Power $(\mathrm{ICC}=.926 ; 95 \% \mathrm{CI}=.897-$

229.947 ) between the Vertec and My Jump. These ICC results indicate that the Vertec and My Jump

230 are highly consistent with each other with respect to measurement of maximum VJ height (Table

231 2, Figure 1A and 1B). Furthermore, given the greater ICC estimate and greater and narrower CI

232 for Peak Power values, our results indicate that the use of calculated Peak Power as the

233 dependent variable resulted in stronger reliability values compared to VJ height. The ICC

234 estimate and 95\% CI demonstrated poor to moderate reliability for jump height depending on

235 sex; males $(n=94)(\mathrm{ICC}=.732 ; 95 \% \mathrm{CI}=.623-.814)$ and females $(=41)(\mathrm{ICC}=.555 ; 95 \% \mathrm{CI}$

$236=.302-.735)$. Consistency for calculated Peak Power values increased for both groups to

237 moderate to excellent; males $(\mathrm{ICC}=.893 ; 95 \% \mathrm{CI}=.844-.928)$ and females $(\mathrm{ICC}=.807 ; 95 \%$

$238 \mathrm{CI}=.667-.892)($ Table 2$)$.

239

240 Agreement

241

242 ICC estimates and 95\% CI demonstrated poor to good reliability for jump height (ICC $=.665$;

$24395 \% \mathrm{CI}=.050-.859)$ and poor to excellent reliability for calculated Peak Power $(\mathrm{ICC}=.851$;

244 CI 95\% .272-.946). Despite reasonable ICC estimates - particularly, for calculated Peak Power

245 - the very broad CI for each dependent variable indicate that the Vertec and My Jump do not

246 consistently produce similar absolute VJ height values relative to each other. A paired-samples

247 -test confirmed the lack of absolute agreement between the tools; mean VJ height using the

248 Vertec $(51.93 \pm 14.36 \mathrm{~cm})$ were found to be significantly higher (statistically) than mean VJ 
249 height values measured using My Jump $(43.05 \pm 12.13 \mathrm{~cm} ; t(134)=12.69, p<0.0001$; Table 2,

250 Figure 2). ICC estimates and 95\% CI demonstrated poor to good reliability for jump height 251 depending on sex; males $(\mathrm{ICC}=.492 ; 95 \% \mathrm{CI}=.086-.774)$ and females $(\mathrm{ICC}=.469 ; 95 \% \mathrm{CI}=$ $252.118-.699)$ and poor to excellent reliability for calculated Peak Power; males (ICC $=.747 ; 95 \%$ $253 \mathrm{CI}=.007-.913)$ and females $(\mathrm{ICC}=.748 ; 95 \% \mathrm{CI}=.425-.881)$. The very broad $\mathrm{CI}$ for each 254 dependent variable indicate that the Vertec and My Jump do not consistently produce similar 255 absolute VJ height values relative to each other for both males and females (Table 2).

256

257 Discussion

258

259

My Jump compared to Vertec demonstrated good to excellent reliability relative to degree of 260 consistency, and poor to excellent reliability relative to absolute agreement. The force plate may 261 be considered the "gold standard" for vertical jump testing accuracy (Menzel et al., 2010), 262 however, this measurement tool is not easily accessible to non-elite and/or non-professional 263 physical activity practitioners due to environmental, financial, time, and/or expertise constraints 264 (and thus, not commonly used by this population). Although preliminary support for the use of My Jump by field practitioners has been established (Balsalobre-Fernández, Glaister \& Lockey, 2015; Gallardo-Fuentes et al., 2016; Driller et al., 2017), these reliability studies have compared My Jump to force plate data. Given that relatively few field practitioners are using force plates to measure vertical jump height and that the goal of applied research is to provide data and recommendations that are likely to be adopted by practitioners, it was important to examine the 270 reliability of My Jump compared to a more commonly used field measurement tool. Like the 271 force plate, the Vertec has also been found to be a reliable vertical jump measurement tool 272 (Klavora, 2000; Caruso et al., 2010; Nuzzo, Anning \& Scharfenberg, 2011), but unlike the force 
273 plate, the Vertec is amenable to multiple testing locations (e.g., laboratory, field, court, etc.) and

274 thus, is more commonly used in "real-world" vertical jump test settings.

275

276 In a similar vein, it was important to examine the reliability of My Jump using a jump that most 277 closely approximates the manner in which individuals actually perform maximum vertical jumps 278 in the real-world. Indeed, previous My Jump reliability studies have employed less ecologically 279 valid jump styles (i.e., CMJ, SJ and DJ) (Balsalobre-Fernández, Glaister \& Lockey, 2015;

280 Gallardo-Fuentes et al., 2016; Driller et al., 2017), thus reducing the generalizability of their 281 findings to the real world. The VJ jump is not without criticism from an experimental control 282 perspective; these criticisms have centered upon two issues: the complexity of the movement, 283 and human measurement error (Luhtanen \& Komi, 1978; Harman et al., 1988; Leard et al., 2007; 284 Menzel et al., 2010; Nuzzo, Anning \& Scharfenberg, 2011).

Reliability relative to absolute agreement between the jump height measurement tools ranged

287 from poor to excellent (Figure $1 \& 2$, Table 2) and the absolute jump height values measured via 288 Vertec were significantly higher than those measured via My Jump (Figure 2, Table 2). Thus, the 289 data from this study indicates that the Vertec and My Jump do not consistently produce similar 290 absolute jump height values relative to each other. These differences are due to the way in which 291 jump height was calculated; the My Jump was based on time in the air and does not account for 292 the upper limb reach component of the jump that was measured by the Vertec (Menzel et al., 293 2010; Nuzzo, Anning \& Scharfenberg, 2011). This finding (a lack of absolute agreement 294 between measurement tools) parallels that found in previous studies examining vertical jump 295 heights in healthy adult participants (Hoffman \& Kang, 2002; Caruso et al., 2010; Menzel et al., 
296 2010). Collectively, based on these findings the recommendation is that field practitioners

297 explicitly use either the My Jump or the Vertec to assess VJ jump height; one tool should be used

298 exclusively for repeat measures and the measurement tools should not be considered

299 interchangeable.

300

301 Reliability relative to degree of both consistency and absolute agreement increased for the

302 calculated Peak Power values compared to jump height measures. The absolute differences in

303 measurements between Vertec and My Jump were smaller when Peak Power was calculated from

304 jump height (Table 2). Peak Power calculations (Sayers et al., 1999) include body mass and

305 body mass significantly affects an individual's ability to jump. Individuals with similar jump

306 heights can have very different Peak Power values due to body mass differences (Harman et al.,

307 1988; Johnson \& Bahamonde, 1996). One limitation of using peak power in the current study,

308 which should be noted, is that the use of the Sayers regression equation to estimate peak power

309 inherently inflated the ICC estimates due to the shared variance of body weight. Thus, the reader

310 may wish to interpret the specific ICC values with caution. However, the reader can still

311 confidently conclude that the use of Peak Power values demonstrated higher reliability values

312 compared to that observed of jump height values.

313

314 From an ecological validity perspective, the specific jump style employed, the use of healthy

315 adult participants from across the general university population, the relatively large number of

316 participants, and our decision to test in the field rather than in a controlled laboratory space all

317 represent strengths of the current study. Such data collection methods increase the

318 generalizability of the current results. A possible limitation of our study was that some

319 participants may not have been familiar with the VJ jump style. If that were the case for some 
320 participants, their resultant jumps may have been inconsistent from jump to jump, or, may not

321 have be representative of their "true" maximum vertical jump height. In this study, we aimed to

322 minimize the influence of this limitation and between jump reliability by providing verbal

323 instructions and physically demonstrating the VJ jump style to participants prior to their VJ jump

324 attempts, as well as by taking each participant's highest VJ of their three jump trials.

325 Furthermore, success in the VJ tests requires a maximal impulse to be applied to the ground 326 resulting in maximal momentum of the body (maximal vertical velocity). The net vertical 327 impulse is highly correlated with external power flow to the ground (Knudson, 2009; Winter et 328 al., 2016) resulting in the misuse of "power" associated with vertical jump success. The vertical 329 jump test provides information about the ability of an individual to jump vertically, and the 330 height of the jump should not be interpreted (through a predictive equation based on the height or 331 the flight time of a jump or a series of jumps) as a true measure of the power an individual can 332 generate but more as a measure of the jumping ability of an individual (Tessier et al., 2013). 333 External power flow is a poor descriptor of performance compared with the impulse that changes

334 velocity (Knudson, 2009; Winter et al., 2016), however we used the Sayer's equation to compare 335 the two methods and used the terminology from that paper "Peak Power".

Conclusions

340 Although Vertec and My Jump were found to be comparable tools for measuring VJ jump height,

341 the relative ease of use, affordability, and portability makes My Jump an attractive option for

342 non-elite and/or non-professional movement practitioners. However, practitioners should be 
343 aware that absolute VJ jump values for Vertec and My Jump, respectively, will differ

344 significantly from each other (although the use of the "peak power" regression equation that

345 includes body weight will minimize this difference). Thus, regardless of whether the practitioner

346 chooses to use Vertec or My Jump, it is recommended that the selected tool should be used

347 exclusively during repeated measures within-subject testing of individuals or groups.

348

349

350

351

352

353

354

355

356

357

358

359

360

361

362

363

364

365

\section{References-}

Aragón LF. 2000. Evaluation of Four Vertical Jump Tests: Methodology, Reliability, Validity, and Accuracy. Measurement in Physical Education and Exercise Science 4:215-228. DOI: 10.1207/S15327841MPEE0404_2.

Ayán-Pérez C., Cancela-Carral JM., Lago-Ballesteros J., Martínez-Lemos I. 2017. Reliability of Sargent Jump Test in 4- to 5-Year-Old Children. Perceptual and Motor Skills 124:39-57. DOI: $10.1177 / 0031512516676174$.

Balsalobre-Fernández C., Glaister M., Lockey RA. 2015. The validity and reliability of an iPhone app for measuring vertical jump performance. Journal of Sports Sciences 33:1574-1579. DOI: 10.1080/02640414.2014.996184.

Bosco C., Luhtanen P., Komi PV. 1983. A simple method for measurement of mechanical power in jumping. European journal of applied physiology and occupational physiology $50: 273-282$.

Buckthorpe M., Morris J., Folland JP. 2012. Validity of vertical jump measurement devices. Journal of Sports Sciences 30:63-69. DOI: 10.1080/02640414.2011.624539. 
366 Burr JF., Jamnik RK., Baker J., Macpherson A., Gledhill N., McGuire EJ. 2008. Relationship of 367 physical fitness test results and hockey playing potential in elite-level ice hockey players. 368 The Journal of Strength \& Conditioning Research 22:1535-1543.

369 Carlos-Vivas J., Martin-Martinez JP., Hernandez-Mocholi MA., Perez-Gomez J. 2016.

370

371 Validation of the iPhone app using the force platform to estimate vertical jump height.

372 The Journal of Sports Medicine and Physical Fitness.

Caruso JF., Daily JS., McLagan JR., Shepherd CM., Olson NM., Marshall MR., Taylor ST. 373 2010. Data reliability from an instrumented vertical jump platform. The Journal of Strength \& Conditioning Research 24:2799-2808.

375 376

377 378 379 380 381 382 383 384 385 386 387 388

Castagna C., Ganzetti M., Ditroilo M., Giovannelli M., Rocchetti A., Manzi V. 2013. Concurrent validity of vertical jump performance assessment systems. The Journal of Strength \& Conditioning Research 27:761-768.

Driller M., Tavares F., McMaster D., O’Donnell S. 2017. Assessing a smartphone application to measure counter-movement jumps in recreational athletes. International Journal of Sports Science \& Coaching 12:661-664. DOI: 10.1177/1747954117727846.

Gallardo-Fuentes F., Gallardo-Fuentes J., Ramírez-Campillo R., Balsalobre-Fernández C., Martínez C., Caniuqueo A., Cañas R., Banzer W., Loturco I., Nakamura FY. 2016. Intersession and intrasession reliability and validity of the My Jump app for measuring different jump actions in trained male and female athletes. The Journal of Strength \& Conditioning Research 30:2049-2056.

Gathercole RJ., Sporer BC., Stellingwerff T., Sleivert GG. 2015. Comparison of the capacity of different jump and sprint field tests to detect neuromuscular fatigue. The Journal of Strength \& Conditioning Research 29:2522-2531. 
389 Glatthorn JF., Gouge S., Nussbaumer S., Stauffacher S., Impellizzeri FM., Maffiuletti NA. 2011.

$390 \quad$ Validity and reliability of Optojump photoelectric cells for estimating vertical jump

391 height. Journal of Strength and Conditioning Research 25:556-560. DOI:

392 10.1519/JSC.0b013e3181ccb18d.

393

394

395

396

397

398

399

400

401

402

403

404

405

406

407

408

409

410

411

Harman EA., Rosenstein MT., Frykman PN., Rosenstein RM., Kraemer WJ. 1988. Estimation of human power output from maximal vertical jump and body mass. ARMY RESEARCH INST OF ENVIRONMENTAL MEDICINE NATICK MA.

Hoffman JR., Kang JIE. 2002. Evaluation of a new anaerobic power testing system. The Journal of Strength \& Conditioning Research 16:142-148.

Janot JM., Beltz NM., Dalleck LD. 2015. Multiple off-ice performance variables predict on-ice skating performance in male and female division III ice hockey players. Journal of sports science \& medicine 14:522.

Janz KF., Letuchy EM., Burns TL., Francis SL., Levy SM. 2015. Muscle Power Predicts Adolescent Bone Strength: Iowa Bone Development Study. Medicine \& Science in Sports \& Exercise 47:2201-2206. DOI: 10.1249/MSS.0000000000000648.

Johnson DL., Bahamonde R. 1996. Power Output Estimate in University Athletes. Journal of Strength \& Conditioning Research 10:161-166.

Klavora P. 2000. Vertical-jump tests: a critical review. Strength \& Conditioning Journal 22:70.

Knudson DV. 2009. Correcting the use of the term "power" in the strength and conditioning literature. The Journal of Strength \& Conditioning Research 23:1902-1908.

Koo TK., Li MY. 2016. A Guideline of Selecting and Reporting Intraclass Correlation Coefficients for Reliability Research. Journal of Chiropractic Medicine 15:155-163. DOI: $10.1016 / \mathrm{j} . \mathrm{jcm} .2016 .02 .012$. 
412 Leard JS., Cirillo MA., Katsnelson E., Kimiatek DA., Miller TW., Trebincevic K., Garbalosa JC. 413 2007. Validity of two alternative systems for measuring vertical jump height. The Journal $414 \quad$ of Strength \& Conditioning Research 21:1296-1299.

415 Luhtanen P., Komi RV. 1978. Segmental contribution to forces in vertical jump. European $416 \quad$ Journal of Applied Physiology and Occupational Physiology 38:181-188.

417 Markovic G., Dizdar D., Jukic I., Cardinale M. 2004. Reliability and factorial validity of squat 418 and countermovement jump tests. The Journal of Strength \& Conditioning Research

420

421

422

423

424

425

426

427

428

429

430

431

432

433

434 $18: 551-555$.

Marques MC., Izquierdo M. 2014. Kinetic and kinematic associations between vertical jump performance and 10-m sprint time. The Journal of Strength \& Conditioning Research 28:2366-2371.

Menzel H-J., Chagas MH., Szmuchrowski LA., Araujo SR., Campos CE., Giannetti MR. 2010. Usefulness of the Jump-and-Reach Test in Assessment of Vertical Jump Performance. Perceptual and Motor Skills 110:150-158. DOI: 10.2466/pms.110.1.150-158.

Mujika I., Santisteban J., Impellizzeri FM., Castagna C. 2009. Fitness determinants of success in men's and women's football. Journal of Sports Sciences 27:107-114. DOI: $10.1080 / 02640410802428071$.

Nuzzo JL., Anning JH., Scharfenberg JM. 2011. The reliability of three devices used for measuring vertical jump height. The Journal of Strength \& Conditioning Research $25: 2580-2590$.

de Salles P., Vasconcellos F., de Salles G., Fonseca R., Dantas E. 2012. Validity and Reproducibility of the Sargent Jump Test in the Assessment of Explosive Strength in Soccer Players. Journal of Human Kinetics 33. DOI: 10.2478/v10078-012-0050-4. 
435 Sayers SP., Harackiewicz DV., Harman EA., Frykman PN., Rosenstein MT. 1999. Cross-

$436 \quad$ validation of three jump power equations. Medicine and Science in Sports and Exercise

437 $31: 572-577$.

438

439

440

441

442

443

444

445

446

447

448

449

450

451

452

453

454

455

456

457

Psychological Bulletin 86:420-428. DOI: 10.1037/0033-2909.86.2.420.

Spiteri T., Binetti M., Scanlan AT., Dalbo VJ., Dolci F., Specos C. 2017. Physical determinants of Division 1 Collegiate basketball, Women's National Basketball League and Women's National Basketball Association athletes: with reference to lower body sidedness. Journal of Strength and Conditioning Research:1. DOI: 10.1519/JSC.0000000000001905.

Stanton R., Wintour S-A., Kean CO. 2017. Validity and intra-rater reliability of MyJump app on iPhone 6s in jump performance. Journal of Science and Medicine in Sport 20:518-523. DOI: 10.1016/j.jsams.2016.09.016.

Stephenson ML., Smith DT., Heinbaugh EM., Moynes RC., Rockey SS., Thomas JJ., Dai B. 2015. Total and Lower Extremity Lean Mass Percentage Positively Correlates With Jump Performance. Journal of Strength and Conditioning Research 29:2167-2175. DOI: 10.1519/JSC.0000000000000851.

Teramoto M., Cross CL., Willick SE. 2016. Predictive Value of National Football League Scouting Combine on Future Performance of Running Backs and Wide Receivers. Journal of Strength and Conditioning Research 30:1379-1390. DOI: 10.1519/JSC.0000000000001202.

Tessier J-F., Basset F-A., Simoneau M., Teasdale N. 2013. Lower-Limb Power cannot be Estimated Accurately from Vertical Jump Tests. Journal of Human Kinetics 38. DOI: 10.2478/hukin-2013-0040. 
458 Walsh MS., Ford KR., Bangen KJ., Myer GD., Hewett TE. 2006. The validation of a portable 459 force plate for measuring force-time data during jumping and landing tasks. Journal of $460 \quad$ Strength and Conditioning Research 20:730.

461 Winter EM., Abt G., Brookes FC., Challis JH., Fowler NE., Knudson DV., Knuttgen HG., Kraemer WJ., Lane AM., Van Mechelen W. 2016. Misuse of “power” and other mechanical terms in sport and exercise science research. The Journal of Strength \& Conditioning Research 30:292-300.

Yingling VR., Webb S., Inouye C., O J., Sherwood JJ. 2017. Muscle power predicts bone strength in Division II athletes. Journal of Strength and Conditioning Research. DOI: 10.1519/JSC.0000000000002222.

468

469

470

471

472

473

474

475

476

477

478

479

480

481

482

483

484

485

486

487

488

Figure and Table Captions:

\section{Table 1:}

Age range and average height and mass of the male $(n=94)$ and female $(n=41)$ participants. Mean (SD)

\section{Table 2:}

Interclass correlation values comparing the consistency and absolute agreement of the My Jump and Vertec for vertical jump height (sm) and peak power (W). Mean (SD)

\section{Figure 1:}

Correlation between My Jump and Vertec. A. vertical jump height (cm) r=0.813 B. Peak power (W) $\mathrm{r}=0.926$.

\section{Figure 2:}

Bland-Altman plots depicting the level of agreement in both A. maximal vertical jump height (cm) and B. calculated Peak Power (W). The majority of points below the line of identity (average values of Vertec and My Jump) confirm the lower values using the My Jump compared to the Vertec. 


\section{Table 1 (on next page)}

Participant characteristics

Age range and average height and mass of the male $(n=94)$ and female $(n=41)$ participants. Mean (SD). 
1 Table 1: Participant Characteristics

2

3 Legend. Age range and average height and mass of the male $(n=94)$ and female $(n=41)$ participants.

4 Mean (SD)

5

Age (years)

Height $(\mathrm{m})$

Male $(n=94)$

Female $(n=41)$

Mass (kg)

$1.77(.08)$

18-39

72.8 (9.9)

$1.67(08)$

63.5 (9.3)

6 


\section{Table 2 (on next page)}

Reliability between My Jump and Vertec

Interclass correlation values comparing the consistency and absolute agreement of the My

Jump and Vertec for vertical jump height (sm) and peak power (W). Mean (SD). 
1 Table 2: Reliability between My Jump and Vertec

2

3 Legend. Interclass correlation values comparing the consistency and absolute agreement of the

4 My Jump and Vertec for vertical jump height $(\mathrm{cm})$ and peak power (W). Mean (SD)

5

6

7

8

9

\begin{tabular}{|c|c|c|}
\hline $\begin{array}{c}\text { My } \\
\text { Jump }\end{array}$ & Vertec & $\begin{array}{c}\operatorname{ICC}(3,1)(95 \% \mathrm{CI}) \\
\text { consistency }\end{array}$ \\
\hline
\end{tabular}

\section{All Participants}

Vertical Jump

Height $(\mathrm{cm})$

$\begin{array}{lcc} & 3974 & 4435 \\ \text { Peak Power }(W) & (1043) & (1144)\end{array}$

Females $(n=41)$
43.05

(12.13)

\subsection{3}

$(14.36)^{*}$
31.52

$(8.22)$

0.813

$.747-.863$

.665

$.050-.859$

0.926

$.897-.947$

.851

$.272-.946$

0.555

$.302-.735$

.469

$.118-.699$

Peak Power (W)

$\begin{array}{cc}2952 & 3238 \\ (684) & (706)^{*}\end{array}$

0.807

$.667-.892$

.748

$.425-.881$

Males $(\mathbf{n}=94)$

Vertical Jump
Height $(\mathrm{cm})$

$\begin{array}{cc}48.07 & 58.43 \\ (9.94) & (10.90)^{*}\end{array}$

0.732

$.623-.814$

.492

$.086-.774$

Peak Power (W)

$\begin{array}{cc}4420 & 4958 \\ (840)^{*} & (874)^{*}\end{array}$

0.893

$.844-.928$

.747

$.007-.913$ 
Figure 1

Correlation between My Jump and Vertec.

A. vertical jump height $(\mathrm{cm}) \mathrm{r}=0.813 \mathrm{~B}$. Peak power $(\mathrm{W}) \mathrm{r}=0.926$.

A

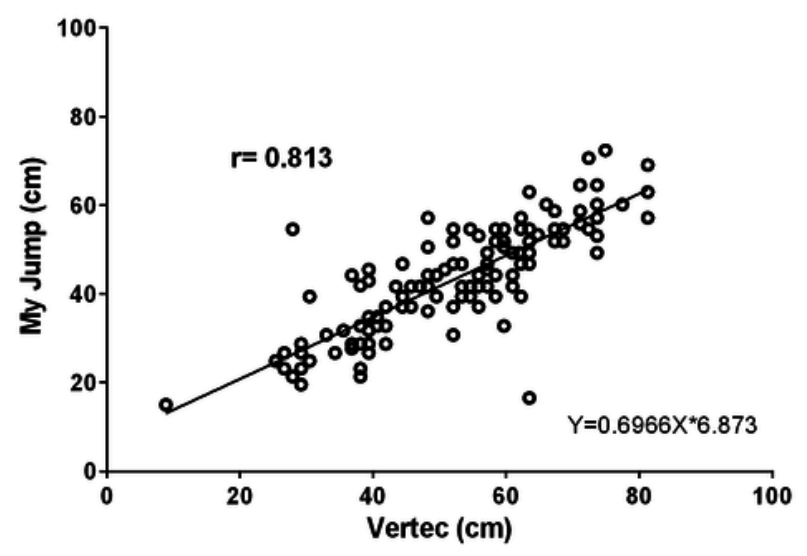

B

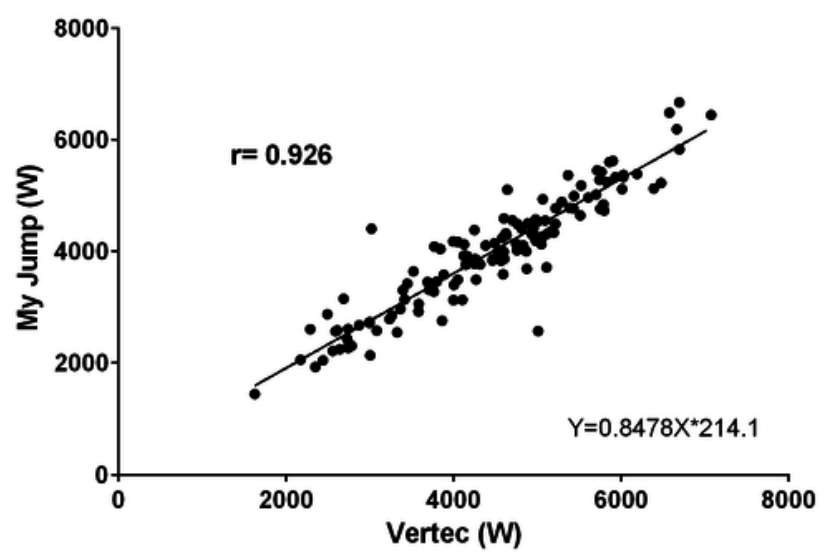


Figure 2

Bland-Altman plots depicting the level of agreement in both A. maximal vertical jump height $(\mathrm{cm})$ and B. calculated Peak Power (W).

The majority of points below the line of identity (average values of Vertec and My Jump) confirm the lower values using the My Jump compared to the Vertec.

A

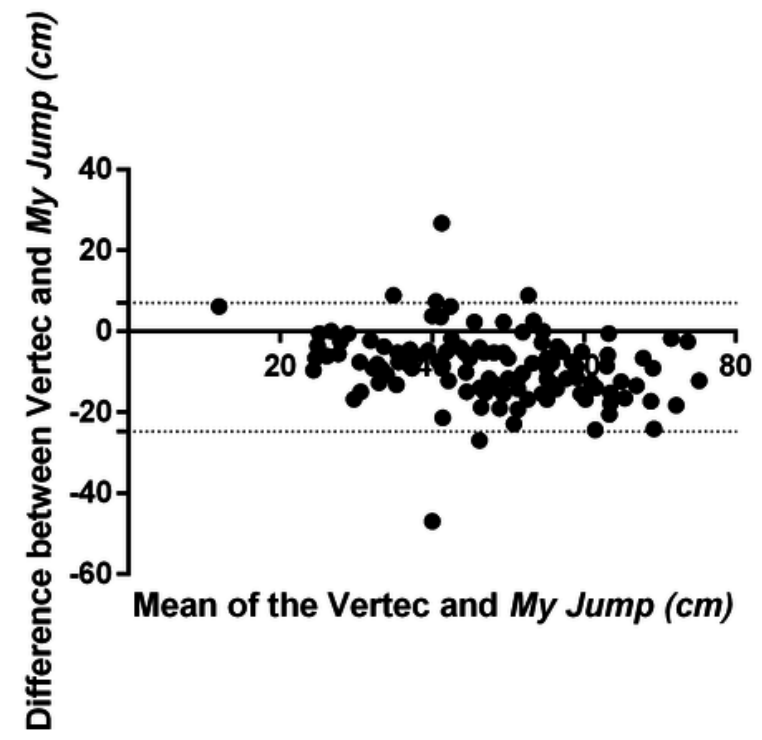

B

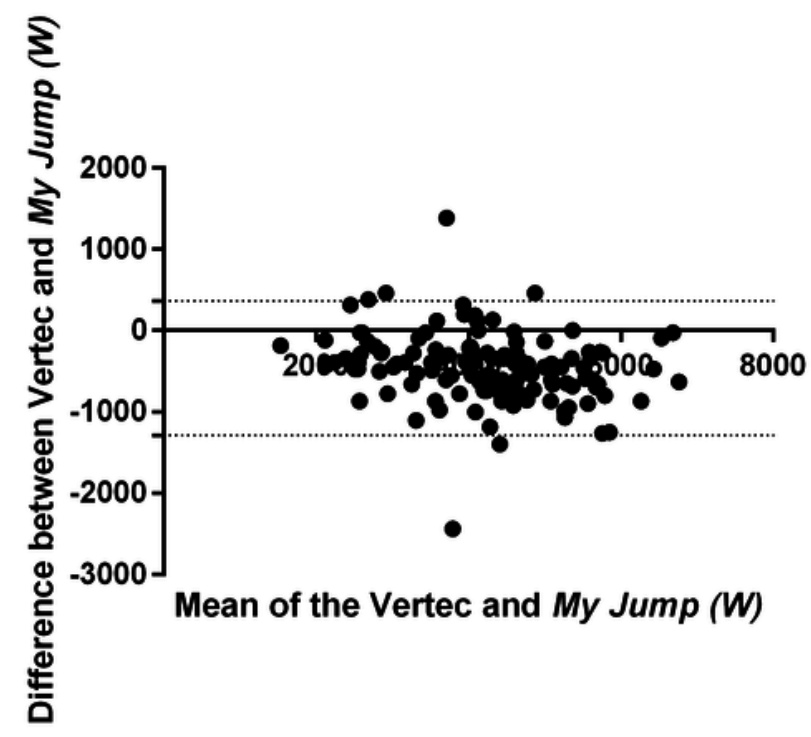

